\title{
Substrate Interaction Domain
}

National Cancer Institute

\section{Source}

National Cancer Institute. Substrate Interaction Domain. NCI Thesaurus. Code C14073.

A Substrate Domain is a protein region that physically interacts stereospecifically, and usually at high affinity, with a specific target substrate. In enzymes, the substrate domain is often, though not necessarily, coincident with the catalytic domain. Typically, substrate interaction results in some protein conformational alteration and functional modification. $(\mathrm{NCl})$ 